\title{
Demographic Effects on Estimates of Automatic Face Recognition Performance
}

\author{
O’ Toole, A. J. \\ Phillips, P. J. \\ An, X. \\ Dunlop J.
}

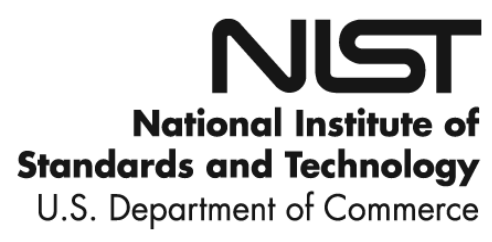


NISTIR 7757

\title{
Demographic Effects on Estimates of Automatic Face Recognition Performance
}

\author{
O’Toole, A. J. \\ Dunlop, J. \\ An, X. \\ U. of Texas at Dallas \\ Phillips, P. J. \\ NIST \\ Information Access Division
}

March 2011

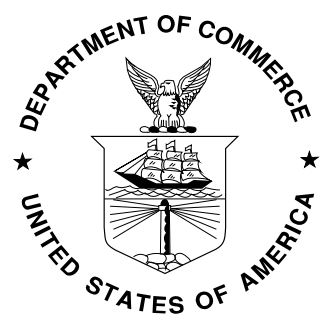

U.S. Department of Commerce Gary Locke, Secretary

National Institute of Standards and Technology

Patrick D. Gallagher, Director 


\section{Demographic Effects on Estimates of Automatic Face Recognition Performance}

\author{
Alice J.O'Toole \\ School of Behavioral and Brain Sciences \\ The University of Texas at Dallas \\ Richardson, TX 75080, USA \\ otooledutdallas.edu \\ Xiaobo An \\ Department of Electrical Engineering \\ The University of Texas at Dallas \\ Richardson, TX 75080, USA \\ xiaobo.anestudent.utdallas.edu
}

\author{
P. Jonathon Phillips \\ National Institute of Standards and Technology \\ Gaithersburg, MD 45435, USA \\ jonathonenist.gov \\ Joseph Dunlop \\ School of Behavioral and Brain Sciences \\ The University of Texas at Dallas \\ Richardson, TX 75080, USA \\ joseph.dunlop@utdallas.edu
}

\begin{abstract}
The intended applications of automatic face recognition systems include venues that vary widely in demographic diversity. Formal evaluations of algorithms do not commonly consider the effects of population diversity on performance. We document the effects of racial and gender demographics on the accuracy of algorithms that match identity in pairs of face images. In particular, we focus on the effects of the "background" population distribution of non-matched identities against which identity matches are compared. The algorithm we tested was created by fusing three of the top performers from a recent US Government competition. First, we demonstrate the variability of algorithm performance estimates when the non-matched identities were demographically "yoked" by race and/or gender (i.e., "yoking" constrains non-matched pairs to be of the same race or gender). We also found a shift in the match threshold required to maintain a stable false positive rate when demographic control scenarios varied. These results were verified with two independent data sets that differed in demographic characteristics. In a second experiment, we explored the effects of progressive increases in population diversity on algorithm performance. We found systematic, but non-general, effects when the balance between majority and minority populations of non-matched identities shifted. Finally, we show that identity match accuracy differs substantially when the non-match identity population varied by race. The results indicate the importance of the demographic composition and modeling of the background population in predicting the accuracy of face recognition algorithms.
\end{abstract}

\section{INTRODUCTION}

The appearance of a face is determined by its gender, race/ethnicity, age, and identity. The goal of most face recognition algorithms is to identify someone as a unique individual. Often this test requires the algorithm to match the identity of faces between images that may vary in the quality or nature of the viewing conditions. The diversity of faces in the real world means that face recognition algorithms must operate over a backdrop of appearance variability that is not related to an individual's unique identity. Thus, face recognition algorithms intended for real-world applications must perform predictably over changes in the demographic composition of the intended application populations. The most likely application sites for algorithms include airports, border crossings, and crowded city sites such as train and metro stations. These locations are characterized by ethnically diverse populations that may vary by the time of year (e.g., tourist season) or even by the time of day (e.g., flights from the Far East arrive in the morning and European flights in the afternoon).

The performance of state-of-the-art automatic face recognition algorithms has been tested extensively over the last two decades in a series of U.S. Government-sponsored tests (e.g., [1]). Measures of algorithm performance in these tests provide the best publicly available information for making decisions about the suitability and readiness of algorithms for applications of importance to security and surveillance. Traditionally, these tests have emphasized measuring performance over photometric conditions such as illumination and image resolution [2], [3], [4], [5]. They have also concentrated primarily on the quality of the "match" (i.e., similarity) between images of the same individuals, considering this to be the critical factor determining algorithm performance. When the degree of similarity between matched identities is high, as is the case when photometric factors are controlled, the algorithm is expected to perform well.

Much less consideration has been given to the distribution against which matched identities are compared. To digress briefly, all measures of the performance of face recognition algorithms rely both on the distribution of data for the population of identity matches (i.e., pairs of images of the same person) and on the distribution of data for nonmatched identities (i.e., pairs of images of different people). By definition, the identity match population contains image pairs of the same race and gender. The non-matched identity population, however, may be structured in several ways. To date, in most evaluations, this distribution consists of pairs of faces that have different identities and which may, or may not, be of different races or genders.

More formally, identity match decisions (same person or different people?) are generally based on a computed simi- 
larity score between two face images. If this score exceeds a threshold similarity score, the faces are judged as an "identity match" (sometimes referred to as an "identity verification"). Otherwise, the images are judged as non-matched identities. False accept errors occur when the computed similarity score between a pair of face images exceeds the match threshold. In most applications, this threshold is set to achieve a low false alarm rate (commonly on the order of 0.001).

As noted, in many formal evaluations of algorithms, the distribution of similarity scores for the non-matched face images includes face pairs that may differ in gender, race/ethnicity, and age [1]. The inclusion of these categorically mismatched image pairs may lead to an over-estimation of an algorithm's ability to discriminate the identity of face pairs. In other words, when non-match face pairs differ on categorical variables such as race and gender, some part of the performance of the algorithms may be due to the easier problem of discriminating faces based on race or gender, than to the more challenging problem of recognizing individual face identities.

In this study, we focus on the problem of how the demographic composition of non-match populations affects estimates of algorithm accuracy. Although demographic variables have been shown to affect both human ([6], [7], [8], [9]) and machine ([10], [11]) accuracy recognizing faces, the effects of these variables in the non-matched identity distributions have not been studied previously. The first goal of this study was to document the effects of yoking nonmatch pairs according to the categorical variables of race and gender, individually, and together. By "yoking" we mean controlling the demographic variables within a non-match pair, such that both faces in the pair are of the same gender, same race, or same gender and race. We also examine the implications of demographic control of the non-match pairs for the choice of a threshold for match/non-match decisions. The second goal was to examine algorithm accuracy with systematic variations in the proportion of a "second" ethnic group in the non-match distribution. Finally, we measured algorithm accuracy when the identity matches were of a particular race and identity mismatches were from another race. This was compared to the case when the match and non-match distributions were of the same race.

\section{ALGORITHM FUSION AND TEST PROTOCOL}

In this section, we overview the algorithm and test protocol common to all experiments. The source of data for these experiments was the FRVT 2006 [1], a U.S. Government sponsored test of face recognition algorithms conducted by the National Institute of Standards and Technology (NIST) (Details of this test and its results can be found elsewhere, [1]). We focused on the uncontrolled-uncontrolled identity match test in the FRVT 2006 (Stimulus Set 1). In that test, algorithms matched identity in pairs of images taken under uncontrolled illumination conditions. To demonstrate the general nature of the problem, we replicated a subset of our results with a second data set (Stimulus Set 2) that
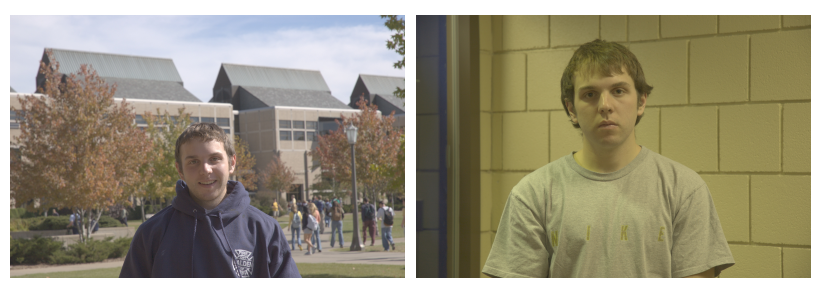

Fig. 1. Example same-identity image pair for the uncontrolled-uncontrolled identity matching task.

TABLE I

SUMMARY DEMOGRAPHIC INFORMATION FOR STIMULUS SET 1.

\begin{tabular}{lrrr}
\hline Ethnicity & Total & Female & Male \\
\hline Caucasian & 716 & 318 & 398 \\
Asian & 264 & 121 & 143 \\
Hispanic & 26 & 10 & 16 \\
Southern Asian & 23 & 2 & 21 \\
Middle Eastern & 6 & 1 & 5 \\
African-American & 12 & 1 & 11 \\
Unknown & 41 & 16 & 21 \\
\hline Total & 1088 & 469 & 619 \\
\hline
\end{tabular}

differed substantially in demographical characteristics from Stimulus Set 1.

1) Stimulus Set 1: The Notre Dame multi-biometric data set [1] was the primary source of face stimuli for the test. This dataset consists of 9,307 images of 570 individuals. The images were photographed with a 6 Mega-pixel Nikon D70 camera and were taken under uncontrolled illumination conditions, either outside or in a corridor or hallway. An example image pair appears in Figure 1 and the relevant demographic breakdown of faces appears in Table I. (Recall that multiple images of individuals are represented in these lists).

2) Stimulus Set 2: The Sandia data set, collected at the Sandia National Laboratory, consists of high-resolution frontal face images taken under both controlled and uncontrolled illumination [1]. The images were taken with a 4 Megapixel Canon PowerShot G2. The average face size for the controlled images was 350 pixels between the centers of the eyes and 110 pixels for the uncontrolled images. For comparison with Stimulus Set 1, the demographic breakdown of faces appears in Table II, with additional detail given to the age of subjects, which is more variable in this data set.

3) Algorithm Test Procedure: In the FRVT 2006 evaluation, each algorithm computed a similarity score for all possible pairs of target and query images. This yielded a matrix of similarity scores where element $s_{i, j}$ represents the similarity between the $i^{t h}$ target and the $j^{t h}$ query image. The goal of the algorithms was to distinguish matched-identity image pairs and non-matched identity image pairs using the similarity scores. The performance of the algorithms was evaluated at NIST using receiver operator characteristic (ROC) curves that plot the proportion of false alarms (false accepts) against the proportion of hits (identity verifications). Because face recognition algorithms are required to operate at low false accept rates, a second measure was also computed. This is 
TABLE II

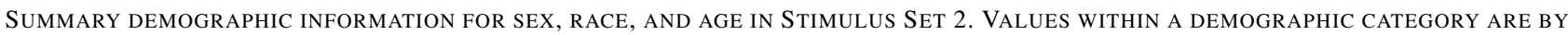
PERCENT (NUMBERS ARE ROUNDED). IF THE NUMBER OF SUBJECTS IN A GIVEN CATEGORY IS LESS THAN 2.5\%, THEN THE CELL IS LEFT BLANK.

\begin{tabular}{|c|c|c|c|c|c|c|c|c|c|c|}
\hline \multirow[b]{2}{*}{ Dataset } & \multicolumn{2}{|c|}{ Sex } & \multicolumn{3}{|c|}{ Race } & \multicolumn{5}{|c|}{ Age } \\
\hline & Female & Male & Caucasian & East Asian & Hispanic & $18-29$ & $30-39$ & $40-49$ & $50-59$ & $60+$ \\
\hline Notre Dame & 62 & 38 & 76 & 13 & & 92 & 7 & & & \\
\hline Sandia & 55 & 45 & 64 & & 21 & 15 & 11 & 23 & 35 & 18 \\
\hline
\end{tabular}

the verification or hit rate at the 0.001 false accept rate. In this paper, we will use both types of measures.

4) Algorithm Fusion Data: The experiments were conducted using data extracted from a fusion of three of the top performing algorithms in the FRVT 2006. The fusion of the similarity matrices operated by first estimating the median and the median absolute deviation (MAD) from 1 in 1023 similarity scores median $_{k}$ and $\mathrm{MAD}_{k}$ reference the median and MAD for the $k^{t h}$ algorithm). The fused similarity scores were computed as the sum of each algorithm's similarity scores after the median has been subtracted and then divided by the MAD. Thus, if $s_{k}$ is a similarity score for the $k^{t h}$ algorithm, and $s_{f}$ is a fusion similarity score, then $s_{f}=$ $\sum s_{k}-\operatorname{median}_{k} / \mathrm{MAD}_{k}$.

The fused data were partitioned subsequently into three performance strata, representing face image pairs that were matched at high, moderate, and low levels of performance. This performance stratification has been referred to elsewhere as the "Good, Bad, and Ugly (GBU) Challenge Problem" [12]. In this study, we used face pairs from the "bad" and "ugly" performance strata, which we will refer to henceforth as the moderately difficult and the difficult face pair conditions. The GBU challenge problem highlights the broad range of algorithm performance when frontal image matches are made. Of note, the pairs of identities used for both the matched and mismatched data were held constant in these strata. Thus, only the images of these identities differed across the three strata. As such, the performance variations among the three levels were due to photometric quality issues (e.g., illumination) or to other extraneous variations in facial expression rather than to recognizability differences of the individual identities.

For any given matched identity pairing (i.e., images of the same person), there were multiple similarity scores available in all three performance strata. We used data from the moderately difficult and difficult face pairs because they had substantial error rates. In each condition, the fused algorithm data consisted of a matrix of similarity scores between all possible pairs of 1088 target face images and 1088 query images. This included 3,306 matched identity pairs and $1,180,438$ non-matched identity pairs. Refer again to Table I for the ethnic and gender composition of the identities in the 1, 088 target and query images used to create the similarity matrix. As Table I makes clear, Caucasians and Asians are the best represented races in the data set. We use only these two races in the experiments we report in Sections IV and $\mathrm{V}$ of this paper. For Section III, which used both Stimulus
Sets 1 and 2, all available races were considered.

\section{DEMOGRAPHIC PAIRING IN NON-MATCH IDENTITY DISTRIBUTIONS}

The goal of these experiments is to document changes in performance estimates for face recognition algorithms as a function of the demographic characteristics of the non-matched identity population. In the first section, we show that performance estimates vary substantially when the non-match population consists of face pairs that are yoked by demographic groups (gender-only, race-only, gender and race). In the second section, we examined the effects of demographic controls on the appropriate choice of a threshold cutoff point for assigning an identity match decision to the face pairs.

\section{A. Partitioning the Performance Estimation Process by De- mographic Bins}

We computed ROC curves in four ways that vary in the demographic controls applied to the non-matched identity distribution. Figure 2 shows the fusion algorithm's performance for the moderately difficult (left) and difficult (right) face pairs. In the No Demographic Matching (No DM) condition, we used all available pairs of mis-matched identity to compute the ROC curve. Thus, non-match pairs could be of the same or different gender and/or race. In the Gender-matched Demographic (DM Gender Only) condition, we used only same-sex pairs to compute the ROC curve, although these pairs could be of a different race. In the Racematched Demographic (DM Race Only) condition, we used only same-race pairs, although these pairs could be of a different gender. In the Gender and Race matched Demographic (DM Gender-Race) condition, the non-matched pairs were of the same-race and same-gender.

The graphs show a substantial decrease in performance as the non-match distribution becomes more demographically controlled. The difference in performance is further seen in Table III, which shows the verification rates at the .001 false alarm rate, as the demographic controls change. These rates change markedly between the condition where there is no demographic control and the condition where both gender and race are controlled. Of note, the verification rate for the difficult face pairs nearly doubles when the demographic controls are removed completely. For the moderately difficult pairs, the verification rate differs by 10 percent between the no control and full control conditions.

Analogous ROC curves for the Sandia data set appear in Figure 2. Similar to the results for Stimulus Set 1, the more 
TABLE III

VERIFICATION RATE AT THE .001 FAlse Alarm RATE With VARIAble TYPES OF DEMOGRAPHIC CONTROL

\begin{tabular}{lrr}
\hline Demographic Control & Moderately Difficult & Difficult \\
\hline No DM & .7925 & .1515 \\
DM Gender Only & .7402 & .1165 \\
DM Race Only & .7408 & .1137 \\
DM Gender and Race & .6851 & .0823 \\
\hline
\end{tabular}

demographically controlled conditions showed decreased performance. This finding bolsters our conclusions about the effects of demographic control using an independent data set with a markedly different demographic structure.

\section{B. Estimating the Decision Threshold Based on Demo- graphic Composition of Non-Matched Identities}

In application-based scenarios, face recognition algorithms use a threshold similarity score to determine if two face images are the same identity or are different identities. Threshold similarity scores are usually chosen to optimize an operational criterion such as a false alarm rate. Typically, thresholds for these automatic face recognition systems are generally set to maintain a false alarm rate of 0.001 . Here we look at the implications of variable demographic controls in the choice of a similarity threshold cutoff for identity match decisions.

In Figure 3, we plot the false accept rate as a function of threshold similarity score using the four demographic control procedures described previously (No DM, DM Gender Only, DM Race Only, DM Gender-Race). (Note that lower more negative values indicate higher similarities.) The left graph shows the function for the moderately difficult pairs and the right graph shows the function for the difficult pairs. The graph below shows the shift for the Sandia data set. In all cases, the threshold that produces a false accept rate of .001 is shifted for the different demographic control conditions. Moreover, the full functions of thresholds are similarly shifted. This indicates that to operate at a particular false alarm rate, threshold values must be set taking into consideration the nature of demographic controls in the non-match population. The results indicate that as the demographic controls tighten, the similarity cutoff threshold shifts to higher similarity scores.

\section{Simulations on Mixed Demographics}

In real world applications, the representativeness of different demographic categories varies arbitrarily in different population contexts, from nearly 100 percent of a single majority race to various degrees of inclusion of (an)other race(s). In this section, we systematically explore the effects of progressive increases in population diversity on algorithm performance. Again, we focus on diversity in the nonmatched identity distribution. We begin by measuring algorithm performance when only one race of faces is included in the non-match identity distribution (Caucasian). Next, we gradually increase the numbers of faces of a second race (Asian) in this distribution and reassess performance.
We refer to this case as the Caucasian-to-Asian condition. In a second simulation, we reversed the process. In this case, we start with only Asians in the non-matched identity distribution, and progressively include more Caucasians. We refer to this case as the Asian-to-Caucsian condition. In the Caucasian-to-Asian condition, the matched identity distribution contained only Caucasian face pairs. In the Asian-to-Causian condition, the matched identity distribution contained only Asian face pairs. (In Section V we explore other combinations of the identity match distributions.)

Because of the imbalance in the number of Caucasian and Asian faces available in the database, these two conditions investigate different scenarios of population shifts. The Caucasian-to-Asian condition simulates a population shift that begins with a single race and ends with a population that is characterized by a strong majority race and moderately sized minority population (slightly over 10 percent). The Asian-to-Caucasian condition simulates a population shift that begins with a single race (Asian) and progressively shifts to equal inclusion of the two races (Asian and Caucasian). As additional Caucasian pairs are included, the population continues to shift toward a majority of Caucasians and a minority Asian population.

\section{A. Methods}

The stimulus set for this experiment included faces of the two races that were best represented in the database: Asians and Caucasians. (See Table I for a listing of numbers of images of people of these races). Note that "Asians" in this study refer to people from the Far East (e.g., China, Japan, Korea). Recall that the original similarity matrix consisted of $1088^{2}$ entries containing the similarity scores for all possible pairs of the images. The data for this experiment consisted of a subset of those similarity scores. Specifically, they were the set of similarity scores from same-gender and same-race Caucasian and Asian face pairs.

Identity Match Data. The identity match data for both conditions did not vary across the simulations. For the Caucasian-to-Asian condition, the similarity scores for all possible pairs of Caucasian identity matches $(n=2110)$ were used to create the identity match distribution. For the Asian-to-Caucasian condition, the similarity scores of all possible pairs of Asian identity matches $(n=866)$ were used to create the identity match distribution. These numbers represent approximately three images of each person in the database, allowing for approximately three pairings of a person with him/herself.

Identity Non-Match Distribution. For the Caucasian-toAsian condition, the simulation began with the non-match distribution consisting of the similarity scores from all possible gender-matched Caucasian pairs of different identities $(n=257,418)$. For the Asian-to-Caucasian case, the simulation began with the non-match distribution consisting of the similarity scores from all possible gender-matched Asian pairs of different identities $(n=34,224)$.

In both cases, face pairs from the other race, were added into the non-match distribution, 1000 at a time, and the 

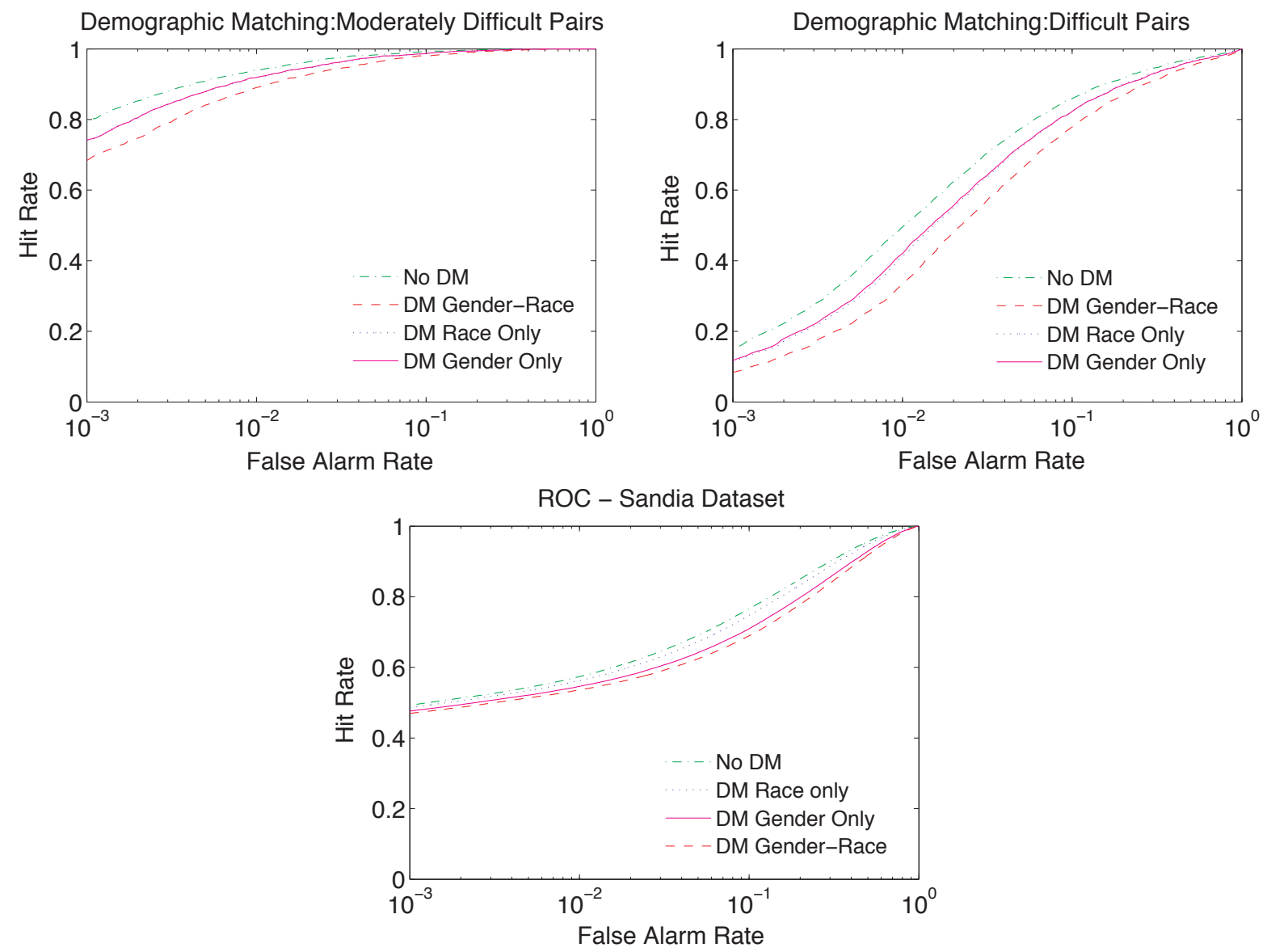

Fig. 2. ROC curves for the moderately difficult (left) and difficult (right) face pairs with different kinds of demographic control on the non-matched identity pairs. Performance is best when the pairs are uncontrolled (No DM) and worst when the pairs are same-gender and same-race pairs (DM Gender-Race). The gender-only and race-only controls yield performance in between the no-control and full control conditions. Analogous ROC curves for the Sandia stimulus set appear in row 2 and support these findings using a database with different demographic characteristics.

verification rate at the 0.001 false alarm rate was computed.

\section{B. Results}

For completeness and clarity, before reporting the results of the demographic mixing in the non-match distribution, we first present the baseline performance of the algorithm for the Asian versus Caucasian face pairs in Figure 4. The top two panels indicate that algorithm performance for the moderately difficult face pairs was marginally better for the Asian faces (left panel). When the face pairs were difficult, the performance was consistently better for the Caucasian faces (right panel). We do not offer a strong interpretation of this finding, because it is arbitrarily dependent on the three algorithms chosen for the fusion. Two of these algorithms were from Western countries and the third algorithm was from a country in East Asia. In the bottom two panels of Figure 4, we see a substantial shift in the similarity threshold that produces a constant false alarm rate when the face race differs. Again this indicates the importance of demographics in the choice of a threshold value chosen to achieve a constant false alarm rate.

The results from the demographic mixing of the non- match distribution appear in Figure 5. The top lines in the figure show the performance for the moderately difficult pairs and the bottom lines show performance for the difficult pairs. The blue lines show verification rate at 0.001 false accept rate as Asian face pairs are added into the non-match distribution for Caucasian face pairs. The red lines show this rate as Caucasian face pairs are added into the non-match distribution for Asian face pairs. As noted, the Caucasian to Asian condition approximates a case where a minority race gradually becomes better represented in the population until it reaches its maximum, in this case at 13.3 percent of the population.

Figure 5 shows that the effect of this gradual increase in representation of the minority race is a steady decline in the verification rate at the .001 false accept rate. The Asian-toCaucasian condition approximates a case where a minority race gradually becomes better represented in the population until it reaches its equilibrium with the first race (50 percent diversity). Ultimately, Caucasians become the majority race (red lines on the graph). Two results are apparent from this simulation. First, in contrast to the Caucasian-to-Asian simulation, which shows a theoretically comparable situation 

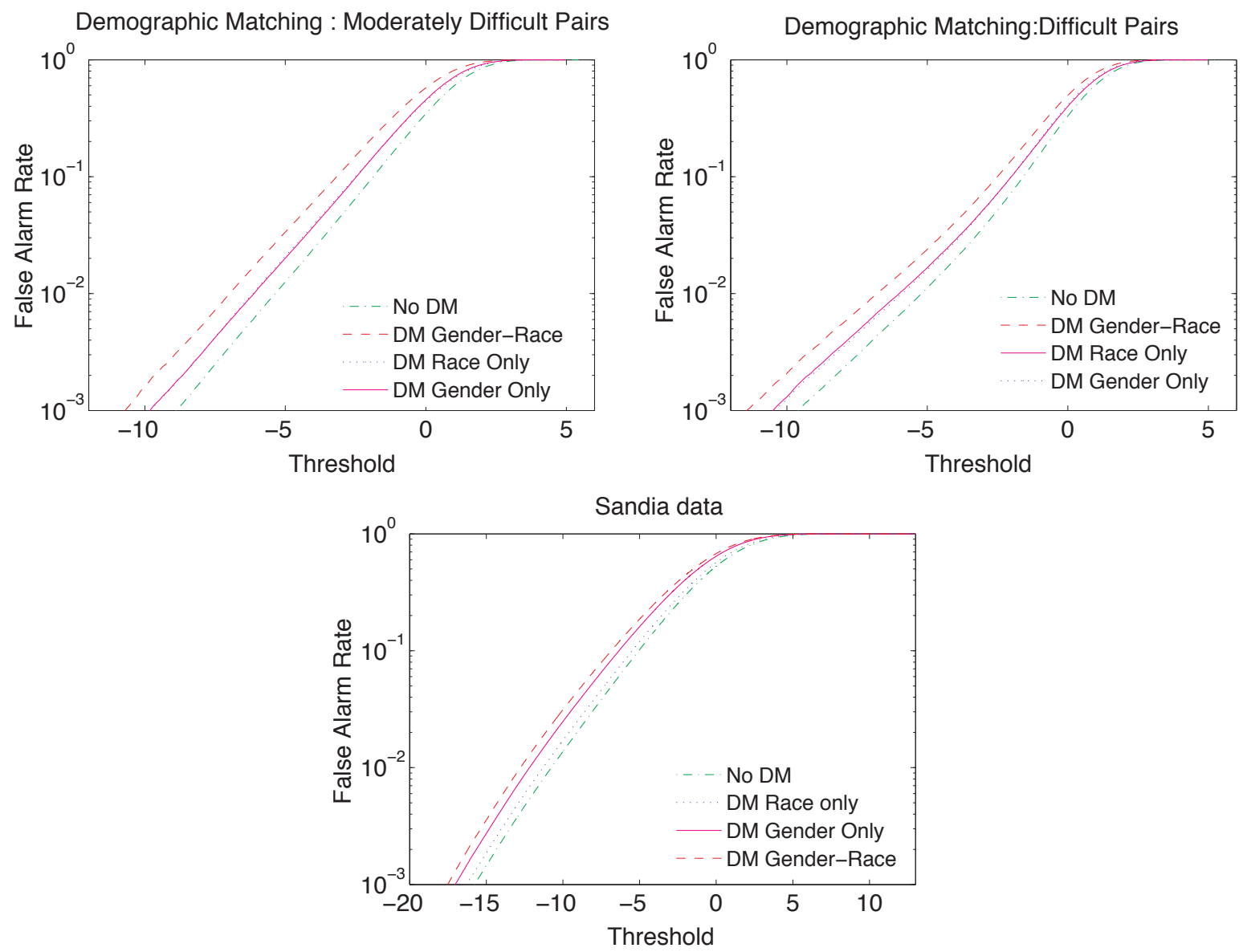

Fig. 3. False alarm rate is plotted as a function of threshold similarity score, using the four demographic control procedures. (Note that lower more negative values indicate higher similarities.) The left graph shows the this function for the moderately difficult pairs and the right graph shows the function for the difficult pairs. The graph below shows analogous data from the Sandia stimulus set. The threshold shifts toward values in the target distribution (i.e., higher similarity scores) as the demographic controls tighten.

up to 13 percent diversity, verification rate increases in this range. This suggests that the effect on verification rate in these situations is not a general result, but rather, is likely to depend both on the percentage diversity and on the algorithm itself, which may process faces of different races in different ways. The second result is the gradual continued increase in verification rate as Caucasians become the majority race in the non-match distribution. Over the full range of diversity percentages, the verification rate at the .001 false alarm rate varies by as much as 0.12 .

\section{Demographically “Reversed” Identity Match AND Mismatch DisTRIBUTIONS}

In the final experiment, we explore the case when the population of face matches to be detected are of a different race than the population against which these matches will be compared.

We compared verification rate at the .001 false alarm rate with the following four combinations of race in the identity match and identity non-match distributions: a.) Caucasians in the match distribution and Caucasians in the non-match distribution (Caucasian-Caucasian); b.) Caucasians in the match distribution and Asians in the non-match distribution (Caucasian-Asian); c.) Asians in the match distribution and Asians in the non-match distribution (Asian-Asian); and d.) Asians in the match distribution and Caucasians in the nonmatch distribution (Asian-Caucasian).

Table IV lists the verification rates at the .001 false accept rate for the four conditions. There was a sharp drop in verification rate from the Caucasian-Caucasian condition to the Caucasian-Asian case. This was true for both the moderately difficult and difficult face pairs. Surprisingly, the inverse pattern was found in comparing the Asian-Asian condition to the Asian-Caucasian case. In this latter case, the verification rate increased when the race of the non-matched identity distribution differed from the race of the matched identity.

An explanation of this might be found in the mean of the similarity distributions for the match and non-matched identity distributions or in the form of the distributions themselves. A careful inspection of our data suggested both factors at work. Differences in the separation of the various match and non-match distribution means explained some, but not all, of the verification rate data in Table IV. An explana- 

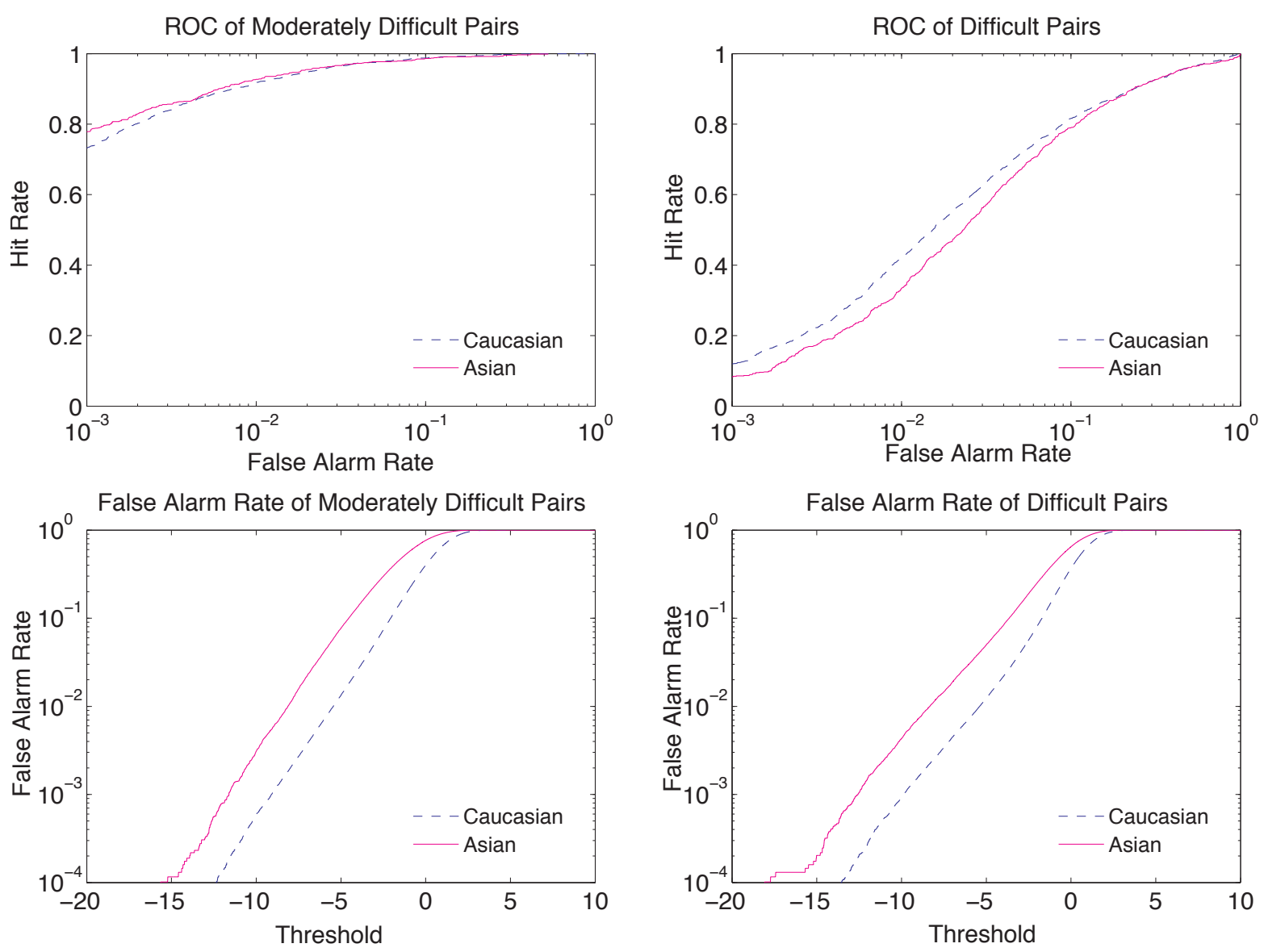

Fig. 4. ROC curves that show the performance of the fusion algorithm on the Caucasian and Asian face pairs (top panels). There is a marginal advantage for Asian faces in the moderately difficult pair condition and an inversion of this pattern for the difficult pairs. Consistent with previous experiments, the bottom panels of the figure show shifts in the similarity threshold functions that produce a constant false alarm rate.

TABLE IV

Verification Rate at the .001 False Alarm Rate With ReVERSED

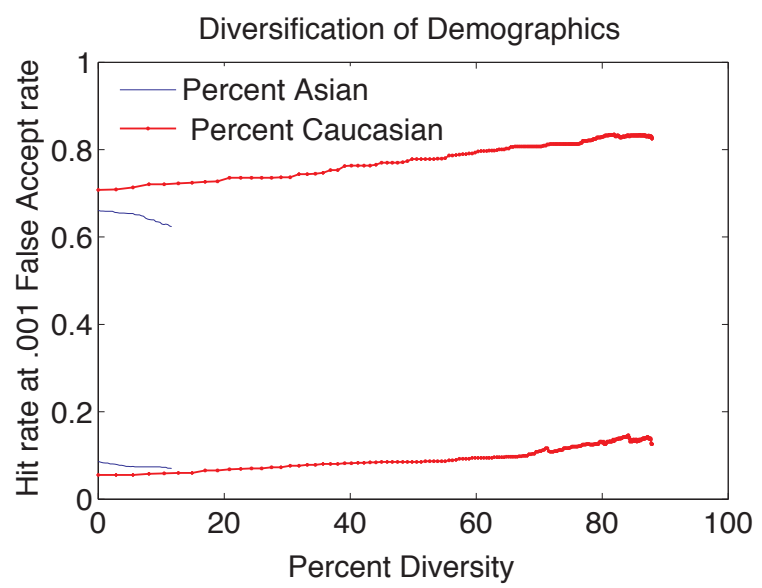

Fig. 5. Verification rate at the 0.001 false accept rate is plotted as a function of the diversification of the the non-match distribution. When the non-match matrix begins with 100 percent Caucasians (blue), verification rate declines as the representation of the Asian minority race increases to its maximum of 13.3 percent. When the non-match matrix begins with 100 percent Asians in the matrix (red), the verification rate increases as the representation of the Caucasians increases, equals Asians, and reverses.

\begin{tabular}{lrrr}
\multicolumn{4}{c}{ DEMOGRAPHICS. } \\
\hline Match & Non-Match & Moderately Difficult & Difficult \\
\hline Caucasian & Caucasian & .66 & .08 \\
Caucasian & Asian & .49 & .03 \\
Asian & Asian & .70 & .06 \\
Asian & Caucasian & .85 & .16 \\
\hline
\end{tabular}

tion of the remaining differences must come, therefore, from subtle aspects of the shapes of the distributions themselves.

\section{CONCLUSIONS}

In summary, all measures of the performance of face recognition algorithms rely both on the distribution of data for identity matches and on the distribution of data for mismatched identities. Traditionally, attempts to improve the performance of face recognition algorithms have emphasized methods that increase the degree of match between images of the same person (e.g., by bridging differences in illumination). Less consideration has been given to the effects of the composition of the non-match identity distributions in producing stable estimates of algorithm performance. These 
estimates are important for predicting how the algorithms will perform in real world environments. In this study, we show that differences in the treatment of demographic diversity in the non-match distribution can radically alter the our estimates of algorithm performance.

The results of this study point to the following factors as determinants of performance. First, the demographic pairing of non-matched identity items can affect both the overall level of performance estimated and the choice of thresholds for match/non-match decisions. If no demographic pairing constraints are imposed, and if the database is diverse, the ability of algorithms to recognize unique identities will be over-estimated. This is because some part of the performance will be based on face categorization (e.g., by gender or race) rather than identity discrimination. Second, we show systematic, but not general, effects on the verification rate at the .001 false alarm rate when a non-match identity distribution increases in racial diversity. Third, again using the measure of verification rate at the .001 false alarm rate, we demonstrate that comparisons of match and nonmatch distributions based on the same and different race of faces can lead to substantial differences in performance expectations.

These second and third findings are particularly troubling because they suggest that it may be difficult to reliably predict algorithm performance without good estimates of the way the match and non-match identity distributions are structured demographically. They further hint at difficulties in choosing operating parameters, such as a threshold match score, that will lead to stable false alarm rates under varying population scenarios. Combined, the present findings pose a new and pressing challenge for this literature to find a method for tuning algorithm performance to the constantly changing demographic environments in which these systems must operate reliably. Again, returning to the context of an airport or tourist attraction, the choice of an appropriate non-match distribution and threshold may have to be reassessed periodically and adjusted as needed. The present study offers the first quantitative evidence on the importance of considering the demographic "background" in setting performance expectations for face recognition systems in the field.

\section{ACKNOWLEDGMENTS}

The authors gratefully acknowledge funding from TSWG/DOD for this work. PJP also thanks the Federal Bureau of Investigation (FBI) for their support of this work. Thanks are also due to Vaidehi Natu for helpful comments on this paper. The identification of any commercial product or trade name does not imply endorsement or recommendation by the National Institute of Standards and Technology or the University of Texas at Dallas.

\section{REFERENCES}

[1] P. J. Phillips, W. T. Scruggs, A. J. O'Toole, P. J. Flynn, K. W. Bowyer, C. L. Schott, and M. Sharpe, "FRVT 2006 and ICE 2006 large-scale results," IEEE Trans. PAMI, vol. 32, no. 5, pp. 831-846, 2010.

[2] R. Gross, S. Baker, I. Matthews, and T. Kanade, "Face recognition across pose and illumination," in Handbook of Face Recognition, S. Z. Li and A. K. Jain, Eds. Sringer, 2005, pp. 193-216.

[3] P. J. Phillips, P. J. Flynn, T. Scruggs, K. W. Bowyer, J. Chang, K. Hoffman, J. Marques, J. Min, and W. Worek, "Overview of the face recognition grand challenge," in IEEE Computer Society Conference on Computer Vision and Pattern Recognition, 2005, pp. 947-954.

[4] P. J. Phillips, H. Moon, S. Rizvi, and P. Rauss, "The FERET evaluation methodology for face-recognition algorithms," IEEE Trans. PAMI, vol. 22, pp. 1090-1104, October 2000.

[5] P. J. Phillips, P. J. Grother, R. J. Micheals, D. M. Blackburn, E. Tabassi, and J. M. Bone, "Face recognition vendor test 2002: Evaluation report," National Institute of Standards and Technology, Tech. Rep. NISTIR 6965, 2003, http://www.frvt.org.

[6] R. S. Malpass and J. Kravitz, "Recognition for faces of own and other race faces," Journal of Personality and Social Psychology, vol. 13, pp. 330-334, 1969.

[7] R. K. Bothwell, J. C. Brigham, and R. S. Malpass, "Cross-racial identification," Personality \& Social Psychology Bulletin, vol. 15, pp. 19-25, 1989.

[8] C. A. Meissner and J. C. Brigham, "Thirty years of investigating the own-race bias in memory for faces: A meta-analytic review," Psychology, Public Policy, and Law, vol. 7, pp. 3-35, 2001.

[9] P. N. Shapiro and S. D. Penrod, "Meta-analysis of face identification studies," Psychological Bulletin, vol. 100, pp. 139-156, 1986.

[10] N. Furl, P. J. Phillips, and A. J. O'Toole, "Face recognition algorithms and the other-race effect: computational mechanisms for a developmental contact hypothesis," Cognitve Science, vol. 26, pp. 797-815, 2002.

[11] P. J. Phillips, F. Jiang, A. Narvekar, and A. J. O'Toole, "An otherrace effect for face recognition algorithms," ACM Trans. Applied Perception, vol. 8, 2010.

[12] P. J. Phillips, J. R. Beveridge, B. A. Draper, G. Givens, A. J. O’Toole, D. Bolme, J. Dunlop, Y. M. Lui, H. Sahibzada, and S. Weimer, "An Introduction to the Good, the Bad, and the Ugly Face Recognition Challenge Problem," in Proceedings, Ninth International Conference on Automatic Face and Gesture Recognition, 2011. 